\title{
Les Italiens et l'image du Maroc dans l'œuvre narrative de Younis Tawfik
}

Isabelle Felici

\section{OpenEdition}

Journals

Édition électronique

URL : http://journals.openedition.org/cei/931

DOI : $10.4000 /$ cei.931

ISSN : 2260-779X

\section{Éditeur}

UGA Éditions/Université Grenoble Alpes

\section{Édition imprimée}

Date de publication : 15 mai 2008

Pagination : 253-264

ISBN : 978-2-84310-121-2

ISSN : 1770-9571

Référence électronique

Isabelle Felici, «Les Italiens et l'image du Maroc dans l'œuvre narrative de Younis Tawfik », Cahiers d'études italiennes [En ligne], 7 | 2008, mis en ligne le 15 novembre 2009, consulté le 01 mai 2019. URL : http://journals.openedition.org/cei/931 ; DOI : 10.4000/cei.931 


\title{
LES ITALIENS ET L'IMAGE DU MAROC \\ DANS L'CEUVRE NARRATIVE DE YOUNIS TAWFIK
}

\author{
Isabelle Felici \\ Université du Sud-Toulon-Var \\ Université Paris 3, CIRCE
}

L'œuvre narrative de Younis Tawfik, qui comporte deux romans, La straniera et La città di Iram, parus en 1999 et 2002 chez l'éditeur milanais Bompiani, se prête particulièrement bien au thème de la différence. La différence y prend d'innombrables formes: elle est linguistique chez cet auteur d'origine irakienne, installé depuis quelques décennies en Italie et qui écrit en italien; elle est culturelle et religieuse, Tawfik étant de culture arabo-musulmane; elle est aussi sexuelle puisque cet homme choisit de mettre en scène des personnages féminins qui sont les narratrices de leur propre histoire. La différence s'insinue aussi dans la forme choisie pour ces romans, pour le premier en particulier, dont la construction, très élaborée, offre au lecteur le double récit des mêmes scènes, une première fois par un narrateur, en fait une image de l'auteur, puis par la narratrice. La différence apparaît enfin sur le plan social et "national», pourrait-on dire, à travers le thème du déplacement: dans le premier roman, celui d'une Marocaine qui arrive clandestinement en Italie, et dans le second, celui d'une Italienne qui effectue un voyage au Maroc.

Le lecteur rencontre donc, dans un jeu complexe de regards qui se croisent $^{1}$, Italiens et Marocains, dans leurs rapports d'autochtones à immigrés à Turin, où se déroule le premier roman, et de touriste à autochtones, à Tanger, Fès, Marrakech, Essaouira - le circuit classique - que traverse la protagoniste du second roman. Ces rencontres et les images transmises au lecteur passent par le filtre du narrateur qu'il est difficile de ne pas assimiler à Tawfik lui-même, lequel reconnait d'ailleurs avoir pris comme point de départ de son premier roman "plus ou moins sa biographie ${ }^{2}$ ». Le récit est ainsi conditionné par le regard d'un non Italien, qui n'est plus tout à fait un étranger puisqu'il s'est déjà parfaitement moulé dans la société

* Notes p. 262

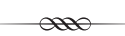

Cahiers d'études italiennes, $\mathrm{n}^{\circ}$ 7, 2008, p. 253-264. 253 
d'accueil. L'image du Maroc, également protagoniste de cette œuvre narrative, passe aussi par le filtre du narrateur, arabe mais non marocain, qui redécouvre la culture arabe à travers le Maroc, alors qu'il est lui-même «privé» de son propre pays depuis son arrivée en Italie en 1979.

Ces deux romans de Tawfik, comme l'ensemble de ses publications et de ses activités culturelles, ont très clairement pour objectif de rapprocher d'un public occidental, en l'occurrence italien, une réalité culturelle arabe et musulmane peu et mal connue, perçue négativement, faussée par le pouvoir médiatique et par les craintes, largement amplifiées par ce même pouvoir médiatique et par certains hommes politiques, que suscite la présence en Italie de migrants qui véhiculent la différence ${ }^{3}$. La lecture que nous proposons de ces romans se fait donc à la lumière de cette approche didactique fréquemment revendiquée ${ }^{4}$ par Younis Tawfik.

Penchons-nous dans un premier temps sur le narrateur, un personnage qui s'entoure d'un silence inquiétant, puisqu'il tait son nom et ne prononce jamais le nom de son pays d'origine, et dont le rôle dans le récit est de servir de pont entre les cultures. Arrêtons-nous sur une description qu'il fait de la maison de son enfance. Pendant quelques pages, le lecteur est transporté dans un monde où l'on dort sur les toits, sur des couvertures posées à même le sol, où l'on peut parfois entrevoir le corps légèrement voilé d'une voisine qui s'est découverte pendant son sommeil. Cette description regorge d'épithètes qui mettent constamment le lecteur à distance, ce qui est compréhensible, mais, ce qui l'est moins, révèle le fossé qui s'est créé entre le narrateur et le décor de son enfance, qualifié d' "arabesco »; l'atmosphère est " orientale», "tipicamente araba et misteriosa» et rappelle parfaitement « $\mathrm{i}$ bellissimi dipinti di Ingres e di Lewis ${ }^{5}$ ». Le narrateur en appelle ainsi aux images, aux stéréotypes, que son lecteur possède déjà sur l'Orient, et semble lui-même avoir adopté ce système de références, celui de l'autre, pour évoquer sa propre culture ${ }^{6}$. Il est en quelque sorte le vecteur d'une vision orientaliste de son propre pays - notons d'ailleurs que Jean-Auguste Dominique Ingres n'a jamais quitté l'Europe - vis-à-vis duquel il montre ainsi un certain détachement. Dans une scène du second roman, qui fait pendant à cette description, on retrouve la même vision extérieure, mais cette fois justifiée, puisque c'est la protagoniste, une Italienne, fraîchement débarquée à Tanger, qui découvre la vision et l'odeur de ce qu'elle est venue chercher au Maroc: l' " exotique ${ }^{7}$ ». Le lecteur des deux romans reçoit donc une description d'un ailleurs par quelqu'un qui se trouve sur le même bord que lui, une touriste et un immigré qui a adopté les critères et les modes de pensée de son pays d'accueil. Le filtre semble définitivement posé. 
Le récit du narrateur de La straniera montre tous les efforts qu'il accomplit pour s'approprier la culture du pays qui l'accueille, privilégiant la fréquentation d'étudiants italiens à l'université, plutôt que des autres étudiants arabes, s'insérant dans la vie sociale italienne, y compris dans le travail : il est devenu architecte, alors que d'anciens camarades d'université ont ouvert une boucherie hallal ou une boîte de nuit à «l'ambiance typiquement orientale». Il n'a des expériences sentimentales et conjugales, d'ailleurs manquées, qu'avec des Italiennes... C'est en fait la rencontre avec une sans-papiers marocaine, Amina, qui provoque un rapprochement vers sa culture d'origine. Amina est un élément déclencheur qui lui fait rechercher une partie de lui-même qu'il avait refoulée, «le passé et le lien avec [sa] terre» (p. 174), qui lui permet de retrouver le plaisir du partage, lorsque, par exemple, ils s'émeuvent en écoutant ensemble les chansons d'Oum Kalthoum, l' «étoile de l'Orient». Amina le fait aussi plonger dans une réalité qu'il n'avait jusque-là pas affrontée, celle des immigrés, de leur «irruption", c'est le mot qu'il emploie, dans une ville, sa ville, auparavant "organisée et propre» et dans laquelle ils créent un «malaise», qu'il ne parvient parfois pas à «tolérer» (p. 131). Lui, qui appartient à la catégorie des «intégrés», reproduit l'attitude et les préjugés de la société italienne envers cette "catégorie de marginaux». S'il a quelques élans de compassion, le rapprochement n'est que provisoire. Il choisit la compagne italienne qui était en concurrence sentimentale avec Amina, laquelle avait pourtant éveillé son "arabité». Il s'éloigne d'elle parce que la clandestinité et sa dépendance envers un mari autoritaire et brutal, auquel elle a voulu échapper, ont fait d'elle une marginale, une prostituée, qu'il ne se sent pas en mesure de "sauver» (p. 133). Cette femme arabe, l'essence de «sa terre", l'a trahi puisqu'elle s'est vendue à d'autres hommes. Empêtré dans deux visions de la réalité entre lesquelles il est incapable de trouver sa propre identité, victime des préjugés des deux cultures, il est condamné à la folie. Le dédoublement de la personnalité est marqué par un changement dans la narration: dans le dernier chapitre de La straniera, le récit est désormais à la deuxième personne du singulier, et le «tu» désigne celui qui avait été jusqu'alors le narrateur. Suicide ou rêve de suicide, le personnage disparaît, en quelque sorte puni parce qu'il n'a pas su préserver ses racines ${ }^{8}$.

Force est de constater qu'Amina est elle aussi punie, par la maladie et la mort, au moment même où elle réussit à sortir de sa condition en trouvant un travail décent et quelqu'un qui l'accueille et l'épaule comme sa propre fille. Elle avait coupé ses cheveux très courts, comme «on les porte maintenant ${ }^{9}$ ", avait changé son prénom, d'Amina en Mina, un procédé auquel 
les "extra-communautaires" se prêtent souvent de mauvais gré, devant l'impossibilité pour des bouches italiennes récalcitrantes de prononcer certains prénoms: Mohamed devient Ali, dans un récit de vie intitulé justement Chiamatemi Alì :

È arrivato il solito problema della pronuncia del mio nome. Mentre i figli e la moglie non hanno difficoltà, il principale non riesce mai a chiamarmi Mohamed: si confonde, pasticcia, si ferma, lo storpia. Ho così sfoderato il mio "secondo nome" (creato appositamente da quando sono in Italia, perché prima non sapevo di averlo): "Chiamatemi Alì!". Così è tutto più semplice ${ }^{10}$.

De la même façon, Khadija devient Caterina, Rabah se transforme en Roberto et, plus étonnant, Younes en John... Fatima semble avoir plus de chance de rester elle-même. En perdant sa chevelure et son nom, Amina/Mina avait commencé à perdre son identité d'où la nécessité, sûrement inconsciente chez l'auteur, de la faire disparaître. Les figures d'immigrés marocains en Italie qui apparaissent dans le second roman, une femme rencontrée par la protagoniste sur la place Jemâa El Fna à Marrakech (p. 64), et un peintre, installé à Essaouira, après une expérience d'émigration clandestine en Italie, où il finit par réussir à suivre des études aux Beaux-Arts (p. 81), sont le reflet inverse d'Amina et connaissent, au lieu du châtiment, le salut et le bonheur car ils ont choisi le retour à la terre/mère.

Ce thème de l'émigration apparaît dans les deux romans, même si c'est de façon périphérique dans La città di Iram, à travers des récits qui mettent en évidence les mécanismes de l'émigration des jeunes Marocains que l'on suit dès avant leur départ du Maroc. C'est d'autant plus efficace, et l'on retrouve là la démarche didactique de Tawfik, que les ouvrages grand public sur ce thème ${ }^{11}$ évoquent rarement la vie des immigrés avant l'émigration $^{12}$. Ce sont aussi des passages importants parce qu'ils sont écrits sans le filtre dont il a été question plus haut. Tawfik nous livre des témoignages qu'il a reçus de façon directe et qu'il transmet en prêtant sa plume à des personnes qu'il a rencontrées, comme la jeune Marocaine dont il a fait la connaissance dans un bar et dont le récit lui a servi pour élaborer le personnage d'Amina ${ }^{13}$; quant aux autres personnages d'immigrés qu'on rencontre dans les deux romans, leur histoire est celle de milliers de personnes, arrivées en Italie avant 1990, clandestinement ou plus souvent avec un visa touristique, et ayant bénéficié de la régularisation qui a accompagné la loi Martelli.

D’une manière générale, ces récits visent à montrer un Maroc que les Italiens ne connaissent guère, ni dans sa réalité sociale, ni même dans sa géographie, une ignorance qui provoque l'agacement des immigrés origi- 
naires de ce pays ${ }^{14}$. Des films comme Marrakech express ${ }^{15}$ ont à ce titre fait plus de dégâts que de bien. Ceux qui n'ont pas visité le pays l'imaginent comme un ailleurs, désertique et sauvage, lointain. Dans l'imaginaire de certains Italiens, il est même assez lointain pour avoir risqué d'être atteint par le raz-de-marée qui a ravagé d'autres régions de la planète à la fin de l'année 2004... Quant aux Italiens qui l'ont visité, ils sont souvent passés à côté d'une réalité sociale ${ }^{16}$ que des mesures policières draconiennes et la "légendaire hospitalité marocaine» contribuent à rendre moins visible, surtout aux yeux de ceux qui ne la cherchent pas.

Par la bouche d'Amina et de quelques personnages secondaires des deux romans, se dessine la réalité que connaissent les jeunes Marocains, hommes et femmes, souvent diplômés, qui ne voient leur salut que dans l'émigration vers l'Europe dont ils rêvent parce qu'ils n'ont pas de travail, ou un travail sous-payé, sans contrat ni protection sociale, rien qui puisse leur permettre de «se construire une vie et un futur ${ }^{17}$ ». À travers le parcours d'Amina, une femme hors normes par sa capacité de révolte, on découvre aussi un Maroc rural en proie à l'analphabétisme et à la misère. Sur ce plan, le contraste avec le deuxième roman est strident. En effet, dans La città di Iram, est au contraire brossée l'image d'un Maroc plus conventionnel, stéréotypé, digne des catalogues des voyagistes. On retrouve le filtre, personnifié par Isabella, la protagoniste du roman, venue au Maroc pour une recherche spirituelle, qui explique le titre du livre: celui-ci fait référence au mythe de la ville d'Iram aux colonnes, auquel s'est intéressé Khalil Gibran et dont il est question dans le Coran ${ }^{18}$. Certains passages du livre de Tawfik ${ }^{19}$ doivent vraisemblablement beaucoup au dialogue métaphysique écrit par Gibran en 1923, intitulé Iram, City of the Lofty Columns ${ }^{20}$. Malgré cette quête mystique, dans laquelle on trouve aussi des échos du roman de Tahar Ben Jelloun, La Nuit sacrée, le lecteur garde surtout l'impression d'un voyage où le matériel et le touristique l'emportent sur le spirituel: on boit beaucoup de thé à la menthe dans des intérieurs marocains riches ou moins riches, dans les ruelles des médinas, au souk de Marrakech et même lors d'une petite escapade en 4 x 4 dans le désert et on rencontre à peu près tout ce que le Maroc a d'exotique aux yeux du touriste ou du voyageur pressé.

Pendant la gestation de ce deuxième roman ${ }^{21}$, s'est produit l'attentat du 11 septembre 2001, que l'auteur a intégré dans la trame narrative sous la forme d'un dialogue entre la protagoniste et une amie marocaine. La scène au cours de laquelle cette jeune musulmane, qui porte le voile, lui raconte les circonstances de l'attentat de New York et les réactions de la jeune Italienne, ramène le lecteur à la question identitaire et au malaise 
déjà perceptible dans le premier roman. Le malaise devient ici manifeste et le filtre évoqué plus haut se transforme en une barrière très nette qui sépare un "vous" d'un «nous» et qui montre les deux systèmes culturels clairement en opposition:

"Temo che voi vogliate la nostra fine, Fatima. Perché ce l'avete con noi? Perché ci odiate così tanto?"

La collera per un attimo mi offuscò la mente e mi fece parlare con rancore a una donna che, come me, non aveva nulla a che fare con ciò che era successo ${ }^{22}$.

Le dialogue entre les cultures dont Tawfik se veut le héraut ${ }^{23}$ semble bien se résumer à une affirmation, de part et d'autre, d'identités monolithiques, inchangeables dans leur substance. "S'ouvrir vers l'autre culture $^{24}$ ", établir un pont, voudrait donc dire donner des clefs pour accéder à la compréhension de l'autre, mais en évitant tout risque de mélange, d'altération. À cette vision substantialiste, qui a des partisans aussi bien chez les nationalistes arabes que chez les adeptes de $L A$ civilisation occidentale, nous opposons une autre conception qui tient compte du caractère hybride et hétérogène des sociétés humaines et de leurs capacités à intégrer les différences sans pour autant les effacer, en renvoyant aux écrits d'Edward W. Said, en particulier à l'essai qu'il rédige en réponse aux critiques qu'a suscitées son ouvrage Orientalisme ${ }^{25}$, et à son ouvrage Culture et impérialisme ${ }^{26}$. Nous renvoyons aussi, en guise d'illustration, au roman Il padre e lo straniero, dont il est question dans ce volume, dans lequel le lecteur assiste réellement à un échange. On voit en effet dans ce roman de Giancarlo De Cataldo un Italien qui n'hésite pas à affronter, quitte à ressentir le malaise qu'il y a à être différent ${ }^{27}$, la "partie arabe» du marché où l'a conduit son ami, un diplomate, bandit, terroriste, agent secret (?) libanais (?), grâce auquel il apprend à aimer son fils handicapé. Il accède aussi grâce à lui à d'autres lieux "étrangers", sans rester en marge, et surtout à un système de pensée dont il s'imprègne et s'enrichit, sans pour autant se "perdre». Il n'y a presque rien de ce "mélange», de cet enrichissement, dans les romans de Tawfik, à l'exception peut-être d'une danseuse orientale $^{28}$, une Italienne que le narrateur prend pour une femme arabe ${ }^{29}$, et de l'enfant conçu par la narratrice du second roman lors de l'escapade dans le désert $^{30}$. Cet enfant, la seule chose qu'Isabella, qui devient Jamila - encore un changement de nom -, semble avoir effectivement ramené de son voyage et de sa quête spirituelle et qu'on voit nourrisson à la fin du roman, a une double identité, au moins génétique, marocaine et italienne, que le lecteur ne voit cependant pas prendre forme dans la réalité.

Il y a d'autant moins d'occasions d'échange culturel dans les deux romans de Tawfik qu'il y a peu de personnages italiens. Dans le second 
roman, la protagoniste est la seule à chercher ce contact, les autres personnages servant surtout à soutenir la trame narrative. Dans La straniera, tous les personnages italiens sont au contraire en contact avec des étrangers, même si c'est de façon superficielle, et entrent dans une sorte de typologie des rapports immigrés/autochtones qui s'instaurent. Parfois, l'étranger est rejeté ou se sent rejeté, comme dans ce restaurant italien où Amina accompagne le narrateur: elle ne voit que des regards désapprobateurs à son égard et beaucoup de froideur, malgré le luxe qui jure avec ses vêtements voyants et son parfum de mauvaise qualité, "typique des jeunes immigrées $^{31}$ ». Mais c'est peut-être dans ce cas un reflet de son propre malaise plutôt que le résultat d'un rejet car la même scène racontée par le narrateur, selon la construction double du roman, ne fait pas allusion à ces regards en coin qui viseraient le couple qu'il forme avec la jeune Marocaine (p. 125 et p. 151). Le rejet est en revanche bien réel de la part d'un commerçant qui répond à Amina, en quête d'un emploi, qu'il n'engage pas de Marocains car "son magasin a sa réputation » à préserver (p. 167). Dans une autre scène du roman, où se déroule un dîner organisé par une collègue de travail du narrateur, Tawfik s'emploie à faire défiler les différentes manifestations d'une xénophobie souvent palpable en Italie: un convive se livre à une classification des nuisances liées aux différentes présences étrangères en Italie, Albanais, Chinois, Arabes... (p. 109), un autre fait remarquer que, tout en n'étant pas hostile aux étrangers, il y a tout de même de quoi avoir peur de sortir la nuit... (p. 111).

C'est aussi une forme de rejet que manifestent les trois compagnes italiennes du narrateur de La straniera, qui lui reprochent d'avoir toujours sa valise prête (p. 39) ou d'être trop absorbé par son passé (p. 173-174) et finissent par le quitter. Ce rejet devient d'ailleurs réciproque étant donné les conclusions que tire le narrateur sur le comportement de la «femme occidentale» (p. 40) en général, avec laquelle toute relation lui semble vouée à l'échec.

D'autres personnages secondaires italiens témoignent au contraire de la solidarité envers les étrangers, comme la commerçante qui prend Amina sous son aile ou la tenancière d'un hôtel qui l'aide dans ses démarches... et beaucoup de compréhension, surtout chez les Italiens qui font ressurgir le passé migratoire de l'Italie, qu'il s'agisse des migrations internes ou de l'émigration proprement dite. Tawfik met en effet en scène plusieurs personnages italiens qui ont vécu directement l'expérience de l'émigration. Sont ainsi évoqués les problèmes identitaires de la première compagne du narrateur, fille de méridionaux installés à Turin et complexée par ses ori- 
gines (p. 37), ou « resservis» les stéréotypes sur les Italiens du sud - ici, une collègue de travail du narrateur d'origine calabraise au très fort caractère ${ }^{32}$ - mais, surtout, Tawfik établit un parallèle entre les deux expériences migratoires que connaît l'Italie qui, de pays d'émigration, est devenue pays d'accueil. Ainsi le propriétaire du bar que fréquente quotidiennement le narrateur affirme-t-il bien comprendre la nostalgie que ressentent les immigrés car il a lui-même quitté son village de Vénétie quand il était enfant et n'a plus personne avec qui échanger des souvenirs ${ }^{33}$. Enfin, lors du dîner entre amis dont il a déjà été question, une convive répond aux propos xénophobes qu'elle entend en rappelant que les Italiens aussi ont été des émigrants, pour certains clandestins ou délinquants ${ }^{34}$.

Cette démarche, très naturelle, de mise en relation du passé migratoire de l'Italie, qu'on a longtemps tendu à refouler, avec les phénomènes migratoires qui touchent le pays depuis trois décennies, est fréquente dans des œuvres à destination du grand public, comme Lamerica de Gianni Amelio ou L'orda. Quando gli albanesi eravamo noi de Gian Antonio Stella ${ }^{35}$, mais aussi dans le domaine de la recherche. C'est le cas sur le terrain de l'histoire sociale en Italie ${ }^{36}$ et au sein de l'italianisme français, chez les chercheurs qui s'occupent des questions migratoires et de leurs implications culturelles ${ }^{37}$. Ces travaux sur l'émigration italienne, parce qu'ils apportent des bémols au mythe de l'intégration facilement réussie des Italiens en France ${ }^{38}$, servent, dans une démarche que l'on pourrait appeler de "civilisation comparée", à mieux comprendre le phénomène actuellement en cours en Italie, ainsi d'ailleurs que les vagues d'immigration plus récentes en France que l'on dit plus visibles.

Tawfik cite lui-même le cas de la France, qui l'intéresse particulièrement pour la fusion linguistique qui s'y pratique:

In Francia, ad esempio, ci sono parole che si usano normalmente e vengono usate da tutti. [...]. L'Italia è un paese ancora giovane per quanto riguarda l'immigrazione, ma il fenomeno è destinato a crescere. Si arriverà dunque a giocare con la lingua, a modellarla come fanno gli scrittori in Francia ${ }^{39}$.

Il prépare quant à lui cette fusion en introduisant dans ses romans, dans le premier surtout, des mots arabes qui ne viennent pas perturber le fil de la lecture, mais au contraire attiser la curiosité du lecteur non arabophone, et en introduisant des poésies, forme d'expression par excellence de la culture arabe ${ }^{40}$, qui viennent rythmer le texte narratif. C'est sans doute sur ce plan linguistique que le rapprochement interculturel voulu par Tawfik est le mieux réussi. Sur le plan narratif, la mise à distance qui apparaît à l'analyse des deux romans ne doit cependant pas être considérée comme un choix définitif de la part de l'auteur, qui donne par ailleurs un 
bon exemple de relation interculturelle réussie, même si elle appartient au passé, à travers les nombreuses pages qu'il consacre à une amitié entre un musulman, le père du narrateur, et un juif dans l'Irak d'avant $1948^{41}$. Peut-être est-ce l'approche didactique qui renforce le filtre déjà perceptible dans La straniera et encore assombri dans le second roman, un filtre qui vraisemblablement empêche l'accomplissement du rapprochement culturel.

S’il y a une perte d'élan littéraire très nettement perceptible entre les deux romans, c'est aussi que Tawfik n'a pas suivi la logique du chemin qu'il avait tracé en entrant dans les lettres italiennes avec La straniera: dans La città di Iram, il a montré un Maroc de pacotille au lieu de creuser celui qu'il a à Turin, à portée de sa main et de sa plume, beaucoup plus intense et authentique. Amina, au lieu de ressusciter, a totalement disparu au profit d'un personnage secondaire du premier roman (Anna-Rita) qui devient la protagoniste du second. Celle-ci, trop préoccupée de sa recherche d'elle-même, est, comme en un raccourci saisissant de $L A$ société occidentale, incapable de s'ouvrir réellement aux autres.

Le premier ouvrage a obtenu un succès public important, 20000 exemplaires vendus avant la parution en format de poche, plusieurs récompenses ${ }^{42}$ et un projet de transposition au cinéma, sans compter l'impact médiatique qui a suivi, car Tawfik se prête volontiers aux interviews des journalistes de la télévision qui l'interrogent sur l'Islam, l'Irak et même sur le rite du thé à la menthe, lui qui connaît plutôt le thé à la cardamome... Tout ceci a bien sûr pesé sur la création, sans compter le poids de la maison d'édition, toujours prête, vu les enjeux économiques, à «monter» de prétendus écrivains qui ne font que «coucher sur le papier leur propre existence et cessent très vite de communiquer quelque chose d'important, si tant est qu'ils l'aient fait la première fois ${ }^{43}$ ". Le phénomène de mode, auquel a vraisemblablement cédé Tawfik, est décrit de façon particulièrement lucide par Mohamed Hmoudane dans son premier roman :

Je compris qu'il fallait peut-être imaginer quelque chose qui collerait bien à mon nom: une terre d'Islam par exemple, avec casbahs et canons rouillés, tels des sexes en berne, muezzins et lumière, blancheur de voile sur fond ocre de muraille, baroque d'ancienne colonie, un port et du désespoir, le désespoir surtout et le large comme promesse. Du "déjà vu" en quelque manière mais en version un peu plus sophistiquée, sans trop forcer sur le folklore ni faire le trop-plein de dévouement dont nous gave une certaine littérature aux effets pervers, bien bonasse et bien pensante teintée d'engagement ${ }^{44}$. 
Sans doute faut-il attendre la prochaine production narrative de Tawfik pour vérifier s'il pourra retrouver la force et l'épaisseur des personnages du premier roman, une force et une épaisseur totalement absentes du second. Quoi qu'il en soit, l'œuvre narrative de Tawfik est entrée dans la littérature sur l'immigration ${ }^{45}$ et a aussi une grande valeur pour le civilisationniste qui se penche sur les questions d'immigration, au même titre que la publicité, la presse, les feuilletons télévisés ou le cinéma, voire la bande dessinée ${ }^{46}$, autant de matériaux qui effraient souvent l'italianisme "classique», toujours prêt à suspecter un glissement "dangereux" vers l'histoire ou les sciences sociales. Une incursion dans ces domaines et une vision interdisciplinaire nous semblent indispensables pour élargir, au moins dans le domaine migratoire qui nous intéresse particulièrement, ce thème de la différence ${ }^{47}$, et pour répondre à la question qui sous-tend forcément toute analyse sur le sujet: combien sommes-nous disposés, de part et d'autre de cette barrière culturelle, d'une brûlante actualité dans la France de l'automne $2005^{48}$, à donner de nous-mêmes et à prendre à l'autre?

\section{Notes}

1. Nous avons traité cet aspect du premier roman de Tawfik dans «Regards croisés sur l'immigration marocaine en Italie. La straniera de Younis Tawfik", Babel, $\mathrm{n}^{\circ} 11$, Revue semestrielle éditée par la Faculté des Lettres et Sciences humaines de l'université du Sud Toulon-Var, premier semestre 2005.

2. Voir l'entretien de Younis Tawfik avec Davide Bregola, "Il machamath tra i Murazzi", juillet 2001, Da qui verso casa, Rome, Edizioni interculturali, 2002, p. 12.

3. Voir C. Marletti (a c. di), Televisione e islam. Immagini e stereotipi dell'islam nella comunicazione italiana, Roma, Nuova ERI, 1995.

4. Voir l'entretien de Younis Tawfik avec Tina Cosmai, «Una cultura di poesia e di fratellanza», <http://caffeeuropa.it/attualita03/168libri-tawfik.html> et le descriptif de son dernier ouvrage, L'Islam dai califfi all'integralismo, Torino, Ananke, 2004.

5. Y. Tawfik, La straniera, Milano, Bompiani Bestesellers, 2003, p. 17, 18 et 23.

6. Voici, à titre de comparaison, comment le narrateur marocain de French Dream, premier roman de Mohamed Hmoudane, décrit le décor qu’a préparé une de ses amies françaises pour leur rencontre: "Dîner aux chandelles, musique douce, dans une atmosphère inspirée de l'Orient, encens, sofa... si bien qu'on se serait cru dans un tableau de la période coloniale de Delacroix. ", M. Hmoudane, French dream, Paris, La différence, 2005.

7. Y. Tawfik, La città di Iram, Milano, Bompiani, 2002, p. 46.

8. Voir l'entretien, déjà cité, de Y. Tawfik avec T. Cosmai.

9. La straniera, op. cit., p. 190.

10. M. Bouchane, Chiamatemi Alì, Milano, Leonardo Paperbacks, 1991, p. 183. Les exemples qui suivent sont eux aussi authentiques.

11. Pour un exemple de ces ouvrages grand public, voir A. Colombo et G. Sciortino, Gli immigrati in Italia, Bologna, Farsi un'idea, Il Mulino, 2004.

12. Ada Lonni (Immigrati, Milano, Mondadori, 2003) adopte au contraire une démarche explicative qui englobe la vie d'avant l'émigration, au moins le contexte politique et économique du pays d'origine. Sur le Maroc, voir p. 54 et suivantes. 
13. Voir l'entretien de Y. Tawfik avec Tiziana Montaldo, "Amore e morte nella Torina multietnica ", décembre 2000, <http://www.volontariperlosviluppo.it/2000_6/00_17.htm>

14. M. Bouchane, Chiamatemi Alì, op. cit., p. 139. Voir aussi p. 85.

15. Marrakech express de Gabriele Salvatores, avec Diego Abatantuono, Columbia Tri Star, 1988.

16. «Anche se abbiamo visitato il loro paese, la nostra cecità raramente ci ha consentito di cogliere la situazione reale in cui versa la popolazione», dit A. Lonni, op. cit., p. 54.

17. La straniera, op. cit., p. 77.

18. Voir la sourate 89.

19. La città di Iram, op. cit., p. 107.

20. Kh. Gibran, Les Dieux de la terre suivi de Iram, cité des Hautes colonnes et de Lazare et sa bien-aimée, Paris, Arthème Fayard, Mille et une nuits, 2003.

21. Voir l'entretien, déjà cité, de Y. Tawfik avec D. Bregola, p. 16.

22. La città di Iram, op. cit., p. 98 et suivantes. Voir aussi p. 103.

23. Voir par exemple l'entretien de Y. Tawfik avec Francesca De Sanctis, «Sono contro Saddam, ma anche contro la guerra", février 2003,

$<$ http://tools.rcs.it/tools/rcslibri/rassegna_stampa/pdf/bompiani/tawfik.pdf>

24. Voir l'entretien, déjà cité, de Y. Tawfik avec F. De Sanctis.

25. E. W. Said, Orientalisme. L'Orient créé par l'Occident, Paris, Seuil, 2005, notamment p. 375 et 376 de la postface.

26. E. W. Said, Culture et impérialisme, Paris, Arthème Fayard/Le Monde diplomatique, 2000.

27. G. De Cataldo, Il padre e lo straniero, Roma, Edizioni e/o, 2004, p. 21. Une première édition, par Manifestolibri, remonte à 1997.

28. Sur la mode de la danse du ventre qui semble faire fureur à Turin, voir le chapitre "Soft power e mezzaluna. Integrazione e danza del ventre», in F. Paci, L'Islam sotto casa. L'integrazione silenziosa, Venezia, Marsilio, 2004, p. 21 et suivantes.

29. La straniera, op. cit., p. 177.

30. Maria Cristina Mauceri commente assez ironiquement cette maternité: «Il messaggio per i lettori sembra essere che la vera natura e spiritualità di una donna si rivela nella procreazione preferibilmente con un compagno esotico.»

<http://www.disp.let.uniroma.it/kuma/RUBRICHE/NOVITA/kuma6-novita-yawfik.htm>

31. "Il suo profumo non è di buona qualità. Comincio a notarlo anche andando in giro per i mercati e nelle strade: sembra che, oramai, sia diventato tipico delle ragazze straniere. ", La straniera, op. cit., p. 123.

32. «E dotata di una forte capacità di convincimento. Non le si può rifiutare niente. Le sue origini calabresi salterebbero fuori subito, dopo un semplice "no". Ė molto cara e simpatica, ma è anche molto permalosa, e guai a farla arrabbiare: sarebbe capace di tenere il muso per giorni», $i b i-$ dem, p. 102.

33. Ibidem, p. 100-101.

34. Ibidem, p. 109

35. G. Amelio, Lamerica, Italie, 1994. G. A. Stella, L'orda. Quando gli albanesi eravamo noi, Milan, Rizzoli, 2002, maintenant en édition économique.

36. Voir par exemple deux numéros de la revue Il calendario del popolo, intitulés «Balie italiane e colf straniere. Migrazioni al femminile nella storia della società italiana» et "Macaronì e vu'cumprà. Emigrazione e immigrazione nella storia della società italiana ", Milano, Teti editore, [1995] 1997.

37. Nous renvoyons au programme de CIRCE, dirigé par J.-Ch. Vegliante, «L'Italie vue d'ici». Voir également "Regards culturels sur les phénomènes migratoires", Babel, n 11, Université du Sud Toulon-Var, premier semestre 2005, qui porte à la fois sur les questions d'émigration et d'immigration.

38. Voir les nombreux travaux de J.-Ch. Vegliante, notamment sur la langue des Italiens en France, dans la bibliographie de CirCE <http://circe.univ-paris3.fr>, et le débat lancé dans "Italiani in Francia: assimilazione e identità a seconda delle generazioni di immigrazione», Itinera. Paradigmi delle migrazioni italiane, a c. di M. Tirabassi, Torino, 2005, p. 251-273. Sur le cas de 


\section{ISABELLE FELICI}

l'accueil des Italiens à Marseille, voir I. Felici, "L’invasion italienne vue par Louis Bertrand. Ribattiamo il chiodo", Babel, nº 1, Université de Toulon et du Var, 1996.

39. Voir l'entretien, déjà cité, de Y. Tawfik avec D. Bregola, p. 17. Notons que ce phénomène s'explique autant, voire davantage par le passé colonial de la France que par les phénomènes migratoires.

40. Voir l'entretien, déjà cité, de Y. Tawfik avec T. Cosmai.

41. La straniera, op. cit., p. 113 à 121.

42. Voir l'entretien, déjà cité, de Y. Tawfik avec D. Bregola, p. 13.

43. "Devo anche aggiungere che ci sono scrittori migranti che non sono scrittori, ma improbabili scrivani della propria esistenza e finiscono subito di comunicare qualcosa di importante (ammesso che lo abbiano fatto la prima volta) e altri che sono montati dalle case editrici di mercato.» «Il gioco dell'incontro. Diagolo intertestuale con Armando Gnisci», D. Bregola, Da qui verso casa, op. cit., p. 154.

44. M. Hmoudane, op. cit., p. 119.

45. Parmi les nombreux outils que l'on peut trouver en ligne, citons la bande de données élaborée à l'initiative d'Armando Gnisci, qui recense les textes des écrivains migrants,

$<$ http://www.disp.let.uniromal.it/basili2001>

Pour des indications bibliographiques sur la littérature de la migration et la littérature sur l'immigration, on pourra consulter aussi <http://www.comune.fe.it/vocidalsilenzio/>

46. Pour un exemple de cette utilisation plurielle (entretiens oraux, littérature et bande dessinée), voir A. Mauri, «Le charme slave. Images littéraires et paralittéraires de femmes slaves émigrées en Italie. Stéréotypes et réalités" in "Regards culturels sur les phénomènes migratoires", Babel, n 11, Université du Sud Toulon-Var, premier semestre 2005.

47. Un thème qui se complète dans presque toutes les manifestations culturelles (littéraires et cinématographiques notamment) celui de la déviance sociale: prostitution, drogue, trafics en tout genre sont souvent irrémédiablement liés aux figures de l'immigré en Italie. Parmi les rares exceptions, citons le très beau film de Vincenzo Marra, Tornando a casa, 2001.

48. Voir le débat sur les violences urbaines et sur ses résonances avec le cas de l'émigration italienne en France lancé par J.-Ch. Vegliante à l'automne 2005, dans le cadre du programme «L'Italie vue d'ici» et mis en ligne sur le site de CIRCE: <http://circe.univ-paris3.fr> 